\title{
EVALUACIÓN DEL USO DE INHIBIDORES DE CORROSIÓN EN EL DECAPADO ÁCIDO DE UNA PLANTA GALVÁNICA
}

\section{Evaluation of the use of corrosion inhibitors in the acid pickling of a galvanic plant}

\author{
iD Héctor Eduardo Sánchez Vargas ${ }^{1}$ (iD Martha Faustina Nápoles García ${ }^{2}$ \\ (iD) María de Lourdes de la Cruz Aragoneses ${ }^{1}$ (iD Fernando Castillo Picón ${ }^{2}$ \\ 'Universidad de Camagüey, Cuba \\ ¿Universidad Nacional Santiago Antúnez de Mayolo. Huaraz, Perú. \\ Correspondencia: \\ Dr. Héctor Eduardo Sánchez Vargas \\ hector.sanchez@reduc.edu.cu
}

\section{RESUMEN}

La protección por recubrimientos constituye el método más empleado de combate anticorrosivo, dado por su efectividad, economía, universalidad y facilidad de aplicación. Sin embargo, la utilización de estos recubrimientos a nivel industrial conlleva un elevado consumo de energía y productos químicos, así como un marcado impacto ambiental. Una de las vías para minimizar los efectos negativos en este tipo de proceso es el uso de inhibidores de corrosión. Este trabajo se propuso el objetivo de mostrar los resultados de una investigación en la cual se realizó una evaluación para la introducción de inhibidores de corrosión en la operación de decapado ácido de un taller galvánico. Como métodos y técnicas de investigación se emplearon la revisión documental, observación, estudios experimentales, además se aplicaron técnicas de modelación matemática y optimización de procesos. Se obtuvieron como principales resultados: la demostración de la factibilidad del uso de formaldehído y la urotropina para las condiciones del taller; se determinó la concentración óptima del inhibidor en la solución ácida del decapado; se concluyó que las combinaciones de ambos inhibidores con el ácido sulfúrico resultan ser las mejores, con el menor costo de decapado para las condiciones óptimas comparada con el resto de las combinaciones ácido-inhibidor.

Palabras clave: evaluación de inhibidores de corrosión, inhibidores de corrosión, uso de inhibidores de corrosión en el decapado ácido

\section{ABSTRACT}

The protection by coatings constitutes the most used method of anticorrosive combat, given its effectiveness, economy, universality and ease of application. However, the use of these coatings at an industrial level entails a high consumption of energy and chemical products, as well as a marked environmental impact. One of the ways to minimize the negative effects in this type of process is the use of corrosion inhibitors. This work had the objective of showing the results of an investigation in which an 
evaluation was carried out for the introduction of corrosion inhibitors in the acid pickling operation of a galvanic workshop. As research methods and techniques, documentary review, observation, experimental studies were used, in addition, mathematical modeling techniques and process optimization were applied. The main results were: the demonstration of the feasibility of the use of formaldehyde and urotropin for the conditions of the workshop; The optimum concentration of the inhibitor in the acid pickling solution was determined; It was concluded that the combinations of both inhibitors with sulfuric acid turn out to be the best, with the lowest pickling cost for optimal conditions compared to the rest of the acid-inhibitor combinations.

Keywords: evaluation of corrosion inhibitors, corrosion inhibitors, use of corrosion inhibitors in acid pickling

\section{INTRODUCCIÓN}

Las características de Cuba como país tropical y el hecho de que el mismo sea una isla largay estrecha con importantes ciudades costeras, propician un ambiente con elevada agresividad desde el punto de vista corrosivo. Aparejado a esto, el desarrollo económico y social de los últimos años, evidenciado por diferentes programas e inversiones en sectores tan importantes como la minería y el turismo, ha incrementado el uso de sistemas y equipos con marcada proporción de metales dentro de su composición. Estas condiciones actuales requieren de métodos de protección cada vez más eficientes, entre los cuales, los recubrimientos son los más generalizados (Domínguez, Castro, Matos, y Hing, 1987).

Los procesos de recubrimiento a metales han sido tradicionalmente altos consumidores de energía y productos químicos, así como fuente importante de residuos de alta agresividad (Carrillo, 2014). Este trabajo ha sido realizado en una de las empresas metalmecánicas del país, la cual cuenta con un taller de galvánica que presta servicios de recubrimiento superficial. Los procesos de recubrimientos que desarrolla el referido taller de galvánica son Fosfatado, Oxidación Alcalina, Cincado y Cromado. Todos ellos, incluyen como una de sus etapas el decapado químico ácido.

En un diagnóstico realizado previamente en la empresa se detecta que el decapado ácido en los procesos de preparación superficial afecta las dimensiones de las piezas en proceso, trayendo consigo un incremento en las producciones defectuosas y un aumento importante en el consumo de productos químicos y energía. La problemática anterior condujo al objetivo de este trabajo, dirigido a evaluar la introducción de inhibidores de corrosión en el decapado ácido empleando técnicas de análisis de procesos para elevar la efectividad del proceso de recubrimiento.

Se denomina inhibidor a cualquier constituyente de una fase cuya presencia no es esencial para que suceda un proceso electroquímico, pero que produce un retardo del mismo, al modificar el estado superficial del material metálico (Uhlig y Revie, 2008). En cierto sentido, un inhibidor puede considerarse como lo opuesto a un catalizador (Buralla, 2012). Por su referido uso como inhibidor en los procesos de decapado ácido (Camejo y Domínguez, 2015) y su disponibilidad, se seleccionaron el formaldehido y la urotropina como inhibidores a evaluar. El comportamiento de un inhibidor se cuantifica normalmente a través de su eficiencia, que se define como el porcentaje de disminución de la velocidad de corrosión por efecto del agregado de una determinada cantidad de inhibidor (Pizarro, 2006).

\section{MATERIALES Y MÉTODOS}

Para la evaluación de los inhibidores formal dehído y urotropina se procedió a determinar la efectividad de los mismos utilizando como referencia la norma cubana NC. 12-04-39. 1987. Inhibidores de corrosión ácida. Métodos de determinación de la capacidad protectora 
durante el decapado ácido de los metales (CEN, 1987).

Los indicadores de la capacidad protectora que refiere la norma son la eficiencia inhibidora $(\eta)$ y el coeficiente de protección $(\wp)$.

Eficiencia inhibidora $\eta: \quad \eta=\frac{V_{0}-V}{V_{0}} 100(\%)$

El Coeficiente de protección $\wp: \quad \wp=\frac{V_{0}}{V}$

donde:

$V_{0}$ y $V$ - Velocidades de corrosión sin inhibidor y con inhibidor respectivamente, todas expresadas en las mismas unidades de medida.

Para determinar experimentalmente la velocidad de corrosión fue seleccionado el método gravimétrico, siendo:

Velocidad de corrosión: $\left(\mathrm{V}_{\mathrm{V}} \mathrm{V}_{0}\right)=\mathrm{DP}=\frac{P_{i}-P_{f}}{A \cdot t_{e}}$

Al aplicar el método gravimétrico, la velocidad de corrosión (DP) se expresa comúnmente en $\mathrm{g} / \mathrm{m}^{2} \mathrm{~h}$. Esta velocidad de corrosión también se puede expresar en mm/año, llamado comúnmente como índice de penetración (Uhlig y Revie, 2008):

Índice de penetración:

(DE) $-D E=\frac{8,76 \cdot D P_{\text {prom }}}{\rho_{\text {metal }}}$

donde:

$P_{\text {i }}$ - Peso de la pieza antes de someterla al decapado (g).

$P_{f}$ - Peso de la pieza después de someterla al decapado (g).

A - Área de la muestra para determinar la velocidad de corrosión $\left(\mathrm{m}^{2}\right)$.

$t_{\mathrm{e}}$ - Tiempo de exposición de la pieza al decapado (h).

$D P$ - Velocidad de corrosión de las muestras de acero $\left(\mathrm{g} / \mathrm{m}^{2} \mathrm{~h}\right)$.

$\mathrm{P}_{\text {metal }}$ - Densidad del metal sometido al decapado ( $\mathrm{kg} / \mathrm{L})$.

Las piezas probetas fueron preparadas superficialmente mediante desengrase, decapado o pulido de acuerdo a la necesidad, se midieron y se realizó una pesada inicial $\left(P_{i}\right)$. Luego se sometieron al medio corrosivo durante el tiempo seleccionado. Posteriormente se extrajeron del baño, se limpiaron superficialmente con una solución de carbonato de sodio para su neutralización y se eliminaron los productos de la corrosión. Después se colocaron en la estufa durante 2 horas a una temperatura entre 140 y $150^{\circ} \mathrm{C}$ y posteriormente fueron colocadas en una desecadora, para luego proceder a pesarlas nuevamente $\left(P_{f}\right)$. Con los valores iniciales y finales de peso se obtuvo la diferencia de peso y se calculó la velocidad de corrosión según la ecuación 3.

La evaluación de los inhibidores se realizó utilizando para el proceso de decapado tres de los ácidos más empleados para este fin (Prado, 2015), los ácidos clorhídrico, sulfúrico y fosfórico. Igualmente, el acero empleado es un acero de aplicación universal como el SAE 1045, uno de los más empleados en la empresa.

El plan experimental contempló primeramente la determinación de la velocidad de corrosión del referido acero en los tres ácidos sin el uso de inhibidor alguno, luego con cada uno de los ácidos y cuatro niveles de concentración de los inhibidores seleccionados, calculando la eficiencia inhibidoray el coeficiente de protección para cada caso. Con los datos obtenidos se ajustó un modelo matemático y posteriormente se obtuvo el valor óptimo de concentración del inhibidor. Finalmente se comprueba si la aplicación de la mejor combinación ácido inhibidor afecta los requisitos de calidad de los cuatro tipos de recubrimiento que se aplican en el taller (fosfatado, oxidación alcalina, cincado y cromado).

El tiempo de exposición de las piezas en el baño de decapado se estableció en $1 / 2$ hora, tiempo cercano al empleado en la práctica productiva.

Para llevar a cabo el plan experimental se planificaron los tres experimentos que se describen a continuación:

Determinación de la velocidad de corrosión del acero SAE 1045, sin el uso de inhibidor, en $\mathrm{HCl}$, $\mathrm{H}_{2} \mathrm{SO}_{4}$ y $_{3} \mathrm{PO}_{4}$. 
La concentración de los ácidos y la temperatura del baño se ajustaron según las recomendaciones de la norma cubana NC. 1203 01: 1981. Sistema de normas para la protección contra la corrosión.

Preparación de las superficies metálicas para la aplicación de los recubrimientos. Requisitos generales (CEN, 1981), o sea, una concentración de $200 \mathrm{~g} / \mathrm{L}$ para los tres ácidos y temperaturas de $30{ }^{\circ} \mathrm{C}$ para el $\mathrm{HCl}$ y $80^{\circ} \mathrm{C}$ para los ácidos sulfúrico y fosfórico.

Cada tratamiento se realizó con tres réplicas para un total de nueve muestras. Para el experimento se utilizan tres cubas de acero inoxidable, todas con un volumen de trabajo de 5 litros.

La velocidad de corrosión sin inhibidor $\left(V_{0}\right)$ se determinó por la ecuación 3.

Determinación de la velocidad de corrosión del acero SAE 1045 en $\mathrm{HCl}_{1} \mathrm{H}_{2} \mathrm{SO}_{4} \mathrm{y} \mathrm{H}_{3} \mathrm{PO}_{4^{\prime}}$ con el uso de dos inhibidores (formaldehído y urotropina) a cuatro concentraciones diferentes.

Las condiciones del experimento son las mismas del anterior.

Definiendo como variable respuesta la velocidad de corrosión y como factores, el tipo de ácido, el tipo de inhibidor y la concentración del inhibidor, se plantea el siguiente diseño factorial, donde se calcula el número de muestras a utilizar como:

$\mathrm{Nm}=\mathrm{n}_{1}{ }^{{ }_{1}} \mathrm{n}_{2}{ }^{{ }^{2}} \mathrm{n}_{3}{ }^{\mathrm{X}_{3}}=3^{1} 2^{1} 4^{1}=24$

donde:

Factores: $x_{1}$ - tipo de ácido, $x_{2}$ - tipo de inhibidor, $x_{3}$ - concentración del inhibidor.

Niveles: $n_{1}-3\left(\mathrm{HCl}, \mathrm{H}_{2} \mathrm{SO}_{4}\right.$ y $\left.\mathrm{H}_{3} \mathrm{PO}_{4}\right), \mathrm{n}_{2}-2$ (formaldehído y urotropina), $\mathrm{n}_{3}-4$ (1, 2, 3 y $4 \mathrm{~g} / \mathrm{L}$ )

Utilizando tres réplicas por tratamiento, el número total de muestras que se utiliza en el experimento es de 72 piezas. Para la preparación y tratamiento posterior de las piezas se procedió de igual forma que el experimento anterior.

Las velocidades de corrosión con inhibidor (V) se determinaron por la ecuación 3 y se promediaron los resultados de las tres réplicas de cada muestra. Para cada caso se determinó la eficiencia inhibidora y el coeficiente de protección según las ecuaciones 1 y 2 .

Evaluación de la influencia de la introducción de los inhibidores en los requisitos de calidad de los recubrimientos que se aplican en el taller.

Este experimento se realizó con las mismas condiciones de los anteriores en cuanto a la etapa de decapado. El tratamiento anterior y posterior de las piezas siguió la tecnología correspondiente a cada tipo de recubrimiento.

Se definieron como variables respuestas a cada uno de los requisitos de calidad de los recubrimientos que se aplican en el taller y como factores, la composición del baño para la concentración de inhibidor óptima determinada (combinación acido - inhibidor) y el tipo de proceso de recubrimiento, se plantea el siguiente diseño factorial, donde se calcula el número de muestras a utilizar como:

$\mathrm{Nm}=\mathrm{n}_{1}{ }^{\mathrm{1}} \mathrm{n}_{2}{ }^{\times 2}=5^{1 *} 4^{1}=20$

donde:

Factores: $x_{1}$ - composición del baño a la concentración óptima del inhibidor, $x_{2}$ - tipo de proceso de recubrimiento.

Niveles:

$\mathrm{n}_{1}$ - $5(\mathrm{HCl}$ - formaldehído, $\mathrm{HCl}$ - urotropina, $\mathrm{H}_{2} \mathrm{SO}_{4}$ - formaldehído, $\mathrm{H}_{2} \mathrm{SO}_{4}$ - urotropina y $\mathrm{H}_{3} \mathrm{PO}_{4}$ - formaldehído). n - 4 (fosfatado, oxidación, cromado y cincado).

La combinación del $\mathrm{H}_{3} \mathrm{PO}_{4}$ con la urotropina fue desechada ya que para la misma no fue posible ajustar un modelo matemático fiable y por tanto no se pudo determinar la concentración óptima de inhibidor.

Utilizando tres réplicas por tratamiento, el número total de muestras que se utiliza en el experimento es de 60 piezas. 


\section{Modelación matemática del proceso de decapado ácido}

La determinación de las velocidades de corrosión (V0 y V) resultó ser la base para la construcción de un modelo económico del proceso de decapado ácido y la determinación de la concentración óptima del inhibidor para costo mínimo de cada combinación ácido-inhibidor.

El modelo matemático para el costo del decapado (CD) está determinado por los consumos de ácido, inhibidor y vapor de calentamiento en la operación de decapado y por el de hidróxido de sodio en la neutralización del ácido en la planta de tratamiento de residuales de la empresa. El resto de los costos asociados al proceso no se tuvieron en cuenta al ser comunes para cada uno de los tratamientos, resultandos irrelevantes en el presente estudio.

$C_{D}=C_{A C}+C_{I N H}+C_{V}+C_{\mathrm{NaOH}}$

donde:

$\mathrm{C}_{\mathrm{AC}}$ - Costo por consumo de ácido

$\mathrm{C}_{\mathrm{INH}}$ - Costo por consumo de inhibidor

$\mathrm{C}_{\mathrm{v}}$ - Costo por consumo de vapor

$\mathrm{C}_{\mathrm{NaOH}}$ - Costo por consumo de hidróxido de sodio en la neutralización del ácido

\section{Costo por consumo de ácido}

El gasto anual de ácido en el proceso de decapado incluye el consumido durante la reacción química con el metal, el que se vierte como ácido agotado luego de la pérdida de actividad del baño y el arrastrado por las piezas tratadas. Esto se expresa matemáticamente como:

$G_{A C}=G_{A C R}+G_{A C V}+G_{A C A}$

donde:

$G_{A C}$ - Gasto anual de ácido ( $\mathrm{kg} /$ año).

$\mathrm{G}_{\mathrm{ACR}}$ - Gasto anual de ácido por su reacción con el metal kg/ año).

$\mathrm{G}_{A C V}$ - Gasto anual de ácido por el vertido de la cuba kg/ año).

$\mathrm{G}_{\mathrm{ACA}}$ - Gasto anual de ácido por arrastres de las piezas kg/ año).

El consumo de ácido por la reacción química se determina como:

$G_{A C R}=m_{A C R} \cdot C_{P D} \cdot N_{D} \cdot C_{C d} \cdot D_{T A}$ donde:

$m_{A C R}$ - Masa de la solución ácida consumida durante el decapado de una pieza (kg/ pieza).

$C_{P D}$ - Cantidad de piezas por dispositivos (piezas/ dispositivos).

$\mathrm{N}_{\mathrm{D}}$ - Número de dispositivos que caben en una cuba en cada ciclo (dispositivos/ ciclo).

$\mathrm{C}_{c d}$ - Cantidad de ciclos de producción diarios (ciclos/ días).

$\mathrm{D}_{\mathrm{TA}}$ - Días de trabajo al año (días/ año).

Para determinar la cantidad de ácido que se consume durante el decapado de una pieza y luego de haber determinado experimentalmente las velocidades de corrosión para los tres tipos de ácido a diferentes concentraciones de inhibidor, se plantea un balance de masa con reacción química en el sistema. Para este caso, tomando como factor de capacidad el área superficial de la pieza (S), el planteamiento de dicho balance para determinar la variación de la masa del metal con el tiempo es el siguiente:

$$
\frac{\mathrm{dmMe}}{\mathrm{dt}}=\mathrm{Ge}_{\mathrm{e}}-\mathrm{G}_{\mathrm{s}}+\mathrm{S} \cdot \mathrm{r}_{\mathrm{ME}}-\mathrm{S} \cdot \mathrm{r}_{\mathrm{ME}}
$$

Como el sistema es cerrado y el metal se consume en la reacción, queda como:

$\frac{\mathrm{dm} \mathrm{Me}}{\mathrm{dt}}=-\mathrm{S} \cdot \mathrm{r}_{\mathrm{ME}}$

Después de integrar y haciendo los arreglos correspondientes, la cantidad de solución ácida gastada durante la inmersión de una pieza en el baño se determina como:

$m_{A C R}=\frac{s \cdot r_{M E} \cdot M M_{A C} \cdot t_{d}}{M M_{M e} \cdot \frac{n e_{M e}}{n e_{A C}} \cdot f p_{A c} \cdot 1000}$

donde:

$\mathrm{S}$ - Área superficial de la pieza crítica $\left(\mathrm{m}^{2}\right)$.

$r_{\text {Me }}$ - Velocidad de corrosión del metal $\left(\mathrm{g} / \mathrm{m}^{2} \mathrm{~h}\right)$.

$\mathrm{MM}_{\mathrm{AC}}$ - Masa molar del ácido en $(\mathrm{g} / \mathrm{mol})$.

$\mathrm{MM}_{\mathrm{Me}}$ - Masa molar del metal en $(\mathrm{g} / \mathrm{mol})$.

$t_{d}$ - Tiempo de decapado (h).

$\mathrm{D}_{T A}$ - Días de trabajo al año.

ne $_{\mathrm{Me}}$ - Moles estequiométricos del metal en la reacción de corrosión (mol Me).

$n_{A C}-$ Moles estequiométricos del ácido en la reacción de corrosión (mol Ac). 
$\mathrm{fp}_{\mathrm{Ac}}-$ Fracción peso del ácido en la solución.

Los moles estequiométricos se deducen en dependencia del tipo de ácido y su reacción con el metal. Para el caso del acero, cuyo componente fundamental es el hierro sería:

$2 \mathrm{HCl}_{\text {(ac) }}+\mathrm{Fe}_{(\mathrm{s})}=\mathrm{FeCl}_{2 \text { (ac) }}+\mathrm{H}_{2 \text { (g) }}$ $\mathrm{H}_{2} \mathrm{SO}_{4(\mathrm{ac})}+2 \mathrm{Fe}_{(\mathrm{s})}=\mathrm{Fe}_{2} \mathrm{SO}_{4(\mathrm{ac})}+\mathrm{H}_{2(\mathrm{a})}$ $2 \mathrm{H}_{3} \mathrm{PO}_{4(\mathrm{ac})}+3 \mathrm{Fe}_{(\mathrm{s})}=\mathrm{Fe}_{3}\left(\mathrm{PO}_{4}\right)_{2(\mathrm{ac})}+3 \mathrm{H}_{2(\mathrm{~g})}$

El gasto anual de ácido por el vertido de la cuba depende de la frecuencia anual de cambio de baño y esta se determina por una relación directa con la velocidad de reacción, la cual depende de la presencia y concentración del inhibidor. De esta forma se determina la frecuencia anual de cambio de baño como:

$$
\mathrm{F}_{\mathrm{C}}=\frac{\mathrm{FCo}_{\mathrm{co}} \cdot \mathbf{r}_{\mathrm{MEiinh}}}{\mathrm{r}_{\mathrm{MEio}}}
$$

donde:

$F_{c o}$ - Frecuencia anual de cambio sin inhibidor (veces/ año).

$r_{\text {MEio }}$ - Velocidad de corrosión del metal sin inhibidor $\left(\mathrm{g} / \mathrm{m}^{2} \mathrm{~h}\right)$.

$r_{\text {Meiinh }}$ - Velocidad de corrosión del metal con inhibidor $\left(\mathrm{g} / \mathrm{m}^{2} \mathrm{~h}\right)$.

El gasto anual de ácido por vertido de la cuba se determina como:

$$
\mathrm{G}_{\mathrm{ACv}}=\frac{\mathrm{V}_{\mathrm{c}} \cdot \mathrm{C}_{\mathrm{sln}} \cdot \mathrm{F}_{\mathrm{C}}}{\mathrm{f}_{\mathrm{AC}}}
$$

donde:

$V_{c}$ - Volumen de la cuba (m3).

$\mathrm{C}_{\text {sln }}$ - Concentración de la solución ácida en la cuba a verter $(\mathrm{g} / \mathrm{L})$.

El gasto anual de ácido por arrastres de solución en las piezas se determina utilizando el volumen específico de arrastre 0,06 L/ $\mathrm{m}^{2}$ (g), valor recomendado por la norma rusa GOST 9.40280, dadas la ubicación de la pieza (Vertical) y la configuración de la misma (compleja).

$G_{A C A}=m_{A C A} \cdot C_{P D} \cdot N_{D} \cdot C_{d} \cdot D_{T A}$ donde $m_{A C A}$ es la masa de ácido que arrastra una pieza.

$\mathrm{m}_{\mathrm{ACA}}=\frac{\mathrm{g} \cdot \mathrm{s} \cdot \mathrm{C}_{\mathrm{sln}}}{1000 \cdot \mathrm{fp}_{\mathrm{AC}}}$

De esta forma, el costo anual por concepto de gasto de ácido se determina como:

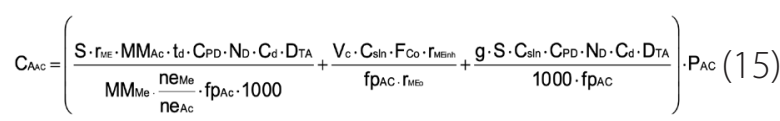

donde:

$\mathrm{P}_{\mathrm{AC}}$ - Precio del ácido $(\$ / \mathrm{Kg})$.

\section{Costo por consumo de inhibidor}

Como teóricamente el inhibidor no se consume en la reacción y solo se gasta por arrastre y con el vertimiento del baño, el gasto anual del mismo se determina como:

$G_{\mathbb{N H}}=G_{I N H V}+G_{I N H A}$

El gasto de inhibidor por el vertido del baño se determina como:

$\mathrm{G}_{\mathrm{INHv}}=\frac{\mathrm{V}_{\mathrm{c}} \cdot \mathrm{C}_{\mathrm{inh}} \cdot \mathrm{F}_{\mathrm{C}}}{\mathrm{fp}_{\mathrm{INH}}}$

$\mathrm{fp}_{\mathrm{INH}}$ - Fracción peso del inhibidor en la solución de formaldehído o en la urotropina.

$\mathrm{C}_{\text {inh }}$ - Concentración de inhibidor en el baño ( $\mathrm{g} /$ $\mathrm{L})$.

$V_{c}$ - Volumen de la cuba de decapado (L)

El gasto de inhibidor por arrastre de las piezas se determina como:

$G_{\mathbb{N} N A}=m_{\mathbb{I N H A}} \cdot C_{P D} \cdot N_{D} \cdot C_{d} \cdot D_{T A}$

donde:

$\mathrm{m}_{\mathrm{INHA}^{-}}$- Masa de inhibidor que arrastra una pieza $(\mathrm{Kg})$.

miNHA $=\frac{\mathrm{g} \cdot \mathrm{s} \cdot \mathrm{C}_{\mathrm{inh}}}{1000 \cdot \mathrm{fp}_{\mathrm{INH}}}$

De esta forma el costo anual por concepto de gasto de inhibidor se determina como:

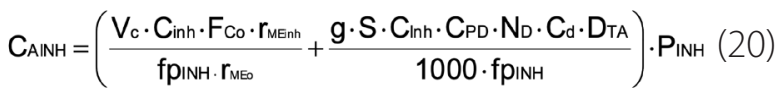


donde $\mathrm{P}_{\mathbb{I N H}}$ es el precio del inhibidor $(\$ / \mathrm{Kg})$.

\section{Costo por consumo de hidróxido de sodio}

El gasto de hidróxido de sodio en la neutralización del ácido en la planta de tratamiento de residuales líquidos industriales de la empresa se puede determinar a partir de los gastos de ácido anuales como:

$$
\mathrm{G}_{\mathrm{NaOH}}=\frac{\mathrm{G}_{\mathrm{AC}} \cdot \mathrm{MM}_{\mathrm{NaOH}} \cdot \frac{\text { ne }_{\mathrm{NaOH}}}{\mathrm{ne}_{\mathrm{Ac}}}}{\mathrm{MM}_{\mathrm{AC}}}
$$

donde:

$\mathrm{MM}_{\mathrm{NaOH}}$ - Masa molar del hidróxido de sodio (g/ $\mathrm{mol})$.

ne $\mathrm{NaOH}_{\mathrm{Na}}$ - Moles estequiométricos del hidróxido en la reacción de neutralización $(\mathrm{mol} \mathrm{NaOH})$.

$n e_{A c}-$ Moles estequiométricos del ácido en la reacción de neutralización (mol ácido).

Calculándose el costo por consumo de hidróxido

$C_{\mathrm{NaOH}}=G_{\mathrm{NaOH}} \cdot P_{\mathrm{NaOH}}$

donde $\mathrm{P}_{\mathrm{NaOH}}$ es el precio del hidróxido de sodio

\section{Costo por consumo de vapor}

Teniendo en cuenta que el vapor llega saturado al área galvánica y que el mismo pudiera condensar totalmente con la instalación de trampas y sistemas de condensado, la energía que entrega será solo por su calor latente de cambio de fase. El balance de energía para determinar la masa de vapor consumido con un $15 \%$ de pérdidas, según refiere la literatura para sistemas como este, resulta:

$\mathrm{Q}_{\mathrm{G}}=0,85 * \mathrm{Q}_{\mathrm{c}}$

donde:

$\mathrm{Q}_{G}$ - Calor ganado por la solución de trabajo (en el baño de decapado)

$\mathrm{Q}_{G}$ - Calor cedido por el vapor

$m_{s} C p_{s o l}\left(T_{t}-T_{a}\right)=0,85 * m_{v}^{*} \Delta H_{f}$

$\mathrm{m}_{\mathrm{s}}=\rho_{\mathrm{sol}} \cdot \mathrm{V}_{\mathrm{c}}$ donde:

$\mathrm{m}_{\mathrm{s}}$ - masa de solución de trabajo $(\mathrm{kg})$.

$\boldsymbol{p}$ sol - Densidad de la solución de trabajo (kg/ L).

$V_{c}$ - Volumen de la cuba $(L)$.

$\mathrm{Cp}_{\text {sol }}$ - Capacidad calorífica de la solución de trabajo ( $\mathrm{kcal} / \mathrm{kg}^{\circ} \mathrm{C}$ ).

$\mathrm{T}_{\mathrm{t}}$ - Temperatura de trabajo $\left({ }^{\circ} \mathrm{C}\right)$.

$\mathrm{T}_{\mathrm{a}}$ - Temperatura ambiente $\left({ }^{\circ} \mathrm{C}\right)$.

$\Delta \mathrm{H}_{\mathrm{f}}$ - Entalpía de cambio de fase del vapor (KJ/

$\mathrm{Kg})$.

$\mathrm{m}_{\mathrm{v}}$ - Masa de vapor $(\mathrm{kg})$.

Teniendo como requisito técnico productivo del proceso que el calentamiento del baño hasta la temperatura de trabajo ocurra en una hora, el consumo de vapor en ese tiempo será determinado como:

$m_{v}=\frac{m_{s} \cdot C p_{s o l}\left(T_{t}-T_{a}\right)}{0,85 \cdot \Delta H_{f}}$

Para calcular el consumo de vapor anual teniendo en cuenta que en las siete horas restantes del turno se consume el $25 \%$ del consumido en la primera hora (para el mantenimiento de la temperatura del baño), y trabajando $D_{T A}$ días al año se llega a la siguiente expresión.

$m_{v}=\left(\left(\frac{m_{s} \cdot C p_{s o l}\left(T_{t}-T_{a}\right)}{0,85 \cdot \Delta H_{f}}\right)+0,25 \cdot 7\left(\frac{m_{s} \cdot C p_{s o l}\left(T_{t}-T_{a}\right)}{0,85 \cdot \Delta H_{f}}\right)\right) D_{T A}(27)$

El costo anual del vapor se determina:

$C_{\text {vap }}=m_{v} \cdot P_{v}$

donde Pv es el precio del vapor $(\$ / \mathrm{kg})$

\section{Determinación de la concentración óptima del inhibidor}

Los datos de velocidades de corrosión obtenidos para cada ácido y concentración de inhibidor se procesaron en una hoja de cálculo de Excel y se obtuvo para cada caso un gráfico que relaciona el costo del decapado con la concentración de inhibidor, luego se ajustó para cada caso un modelo representado por una ecuación polinomial de segundo orden. Este polinomio resultó ser la función objetivo utilizada para la minimización del costo del proceso de decapado. Al aplicar el método analítico de optimización para un problema unidimensional (Boizán, 1988) el mismo se derivó e igualó a cero para 
obtener la concentración óptima de inhibidor a usar en el proceso. Al evaluar la función objetivo para la concentración óptima del inhibidor se obtiene el costo mínimo del decapado ácido por concepto de los gastos concebidos en el modelo económico (Ecuación 5).

\section{RESULTADOS}

Los resultados del experimento dirigido a determinar las velocidades de corrosión sin el uso de inhibidores, se muestran en la tabla 1. Los resultados del segundo experimento, dirigido a determinar las velocidades de corrosión en el decapado ácido usando inhibidores, se muestran en la tabla 2. Los valores de DP, DE, $\eta$ y $y$ representan los valores promedios calculados para las tres réplicas utilizadas para cada tratamiento.
Los resultados de la optimización se muestran en la figura 1. En los modelos polinomiales que se observan en esta figura, la letra " $x$ " (variable independiente) representa la concentración del inhibidor y la letra " $y$ " representa la variable respuesta que es el costo del proceso de decapado $\left(C_{D}\right)$.

La ejecución del tercer experimento se realizó luego de determinada la concentración óptima de inhibidor. Como resultado de este experimento se pudo comprobar que para cada una de las combinaciones ácido - inhibidor a la concentración óptima probada como solución decapante en los procesos de recubrimientos del taller, se cumplieron los requisitos de calidad establecidos para cada uno de los tipos de recubrimientos

\section{Tabla 1}

Comportamiento de la velocidad de corrosión sin inhibidor

\begin{tabular}{|c|c|c|c|c|c|c|}
\hline Ácido & $\begin{array}{c}\mathrm{N}^{\circ} \text { de } \\
\text { muestra }\end{array}$ & $P_{i}(g)$ & $P_{f}(g)$ & $D P\left(g / m^{2} h\right)$ & $D P_{\text {prom }}\left(g / m^{2} h\right)$ & $D E_{\text {prom }}(\mathrm{mm} / \mathrm{año})$ \\
\hline & 1 & 7,118 & 4,989 & 1517,557 & & \\
\hline \multirow[t]{3}{*}{$\mathrm{H}_{3} \mathrm{PO}_{4}$} & 2 & 7,110 & 4,801 & 1645,861 & 1531,575 & 1742,416 \\
\hline & 3 & 7,294 & 5,286 & 1431,308 & & \\
\hline & 4 & 7,056 & 6,997 & 42,055 & & \\
\hline \multirow[t]{3}{*}{$\mathrm{HCl}$} & 5 & 7,014 & 6,947 & 47,758 & 45,619 & 51,899 \\
\hline & 6 & 7,225 & 7,156 & 49,183 & & \\
\hline & 7 & 6,925 & 3,822 & 2211,827 & & \\
\hline \multirow[t]{2}{*}{$\mathrm{H}_{2} \mathrm{SO}_{4}$} & 8 & 6,880 & 3,570 & 2359,377 & 2363,654 & 2689,040 \\
\hline & 9 & 7,155 & 3,620 & 2519,758 & & \\
\hline
\end{tabular}


Tabla 2

\section{Comportamiento de la velocidad de corrosión con inhibidor}

\begin{tabular}{|c|c|c|c|c|c|c|c|c|c|c|c|c|}
\hline \multirow[t]{2}{*}{$\begin{array}{l}\text { Concen. de } \\
\text { inhibidor } \\
(\mathrm{g} / \mathrm{L})\end{array}$} & $\begin{array}{c}\text { DP } \\
\left(\mathrm{g} / \mathrm{m}^{2} \mathrm{~h}\right)\end{array}$ & $\begin{array}{c}\mathrm{DE} \\
\text { (mm/año) }\end{array}$ & $\eta(\%)$ & $\gamma$ & $\begin{array}{c}\text { DP } \\
\left(\mathrm{g} / \mathrm{m}^{2} \mathrm{~h}\right)\end{array}$ & $\begin{array}{c}\mathrm{DE} \\
\text { (mm/año) }\end{array}$ & $\eta(\%)$ & $\gamma$ & $\begin{array}{c}\text { DP } \\
\left(\mathrm{g} / \mathrm{m}^{2} \mathrm{~h}\right)\end{array}$ & $\begin{array}{c}\mathrm{DE} \\
\text { (mm/año) }\end{array}$ & $\eta(\%)$ & $\gamma$ \\
\hline & \multicolumn{4}{|c|}{$\mathrm{H}_{3} \mathrm{PO}_{4}$ - Formaldehído } & \multicolumn{4}{|c|}{$\mathrm{HCl}$ - Formaldehído } & \multicolumn{4}{|c|}{$\mathrm{H}_{2} \mathrm{SO}_{4}-$ Formaldehído } \\
\hline 1 & 1062,789 & 1209,095 & 30,608 & 1,441 & 29,225 & 33,248 & 35,938 & 1,561 & 1379,390 & 1569,280 & 41,642 & 1,714 \\
\hline 2 & 932,702 & 1061,100 & 39,102 & 1,642 & 26,136 & 29,734 & 42,708 & 1,745 & 1132,057 & 1287,898 & 52,106 & 2,088 \\
\hline 3 & 847,285 & 963,924 & 44,679 & 1,808 & 17,107 & 19,462 & 62,500 & 2,667 & 822,282 & 935,479 & 65,211 & 2,875 \\
\hline \multirow[t]{2}{*}{4} & 1049,246 & 1193,687 & 31,492 & 1,460 & 18,824 & 21,416 & 58,728 & 2,423 & 438,712 & 499,107 & 81,439 & 5,388 \\
\hline & \multicolumn{4}{|c|}{$\mathrm{H}_{3} \mathrm{PO}_{4}$ - Urotropina } & \multicolumn{4}{|c|}{$\mathrm{HCl}$ - Urotropina } & \multicolumn{4}{|c|}{$\mathrm{H}_{2} \mathrm{SO}_{4}-$ Urotropina } \\
\hline 1 & 1530,387 & 1741,064 & 0,078 & 1,001 & 16,394 & 18,651 & 64,062 & 2,783 & 575,489 & 654,712 & 72,301 & 3,610 \\
\hline 2 & 943,751 & 1073,670 & 38,380 & 1,623 & 22,810 & 25,950 & 50,000 & 2,000 & 646,310 & 735,283 & 68,892 & 3,215 \\
\hline 3 & 1227,090 & 1396,014 & 19,881 & 1,248 & 18,533 & 21,084 & 59,375 & 2,461 & 507,236 & 577,063 & 75,586 & 4,096 \\
\hline 4 & 1103,775 & 1255,723 & 27,932 & 1,388 & 20,315 & 23,111 & 55,469 & 2,246 & 355,998 & 405,005 & 84,938 & 6,639 \\
\hline
\end{tabular}

$\mathrm{H}_{3} \mathrm{PO}_{4}$ - Formaldehído

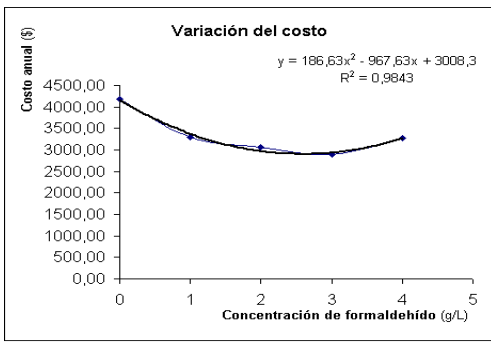

Concentración óptima de inhibidor: 2,59 g/L Costo mínimo de decapado: 2903,34 \$/año

$\mathrm{H}_{3} \mathrm{PO}_{4}$ - Urotropina

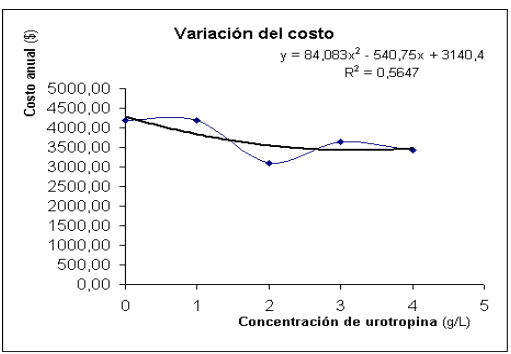

Concentración óptima de inhibidor: Costo mínimo de decapado:

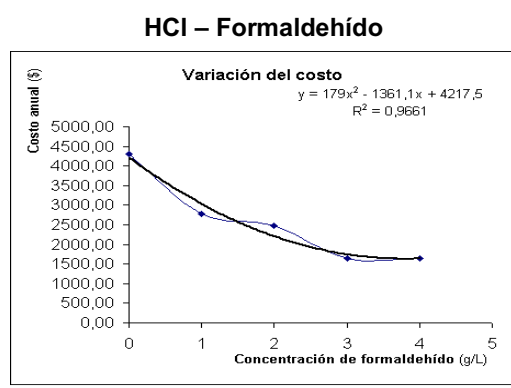

Concentración óptima de inhibidor: $3,80 \mathrm{~g} / \mathrm{L}$ Costo mínimo de decapado: 1630,35 \$/año

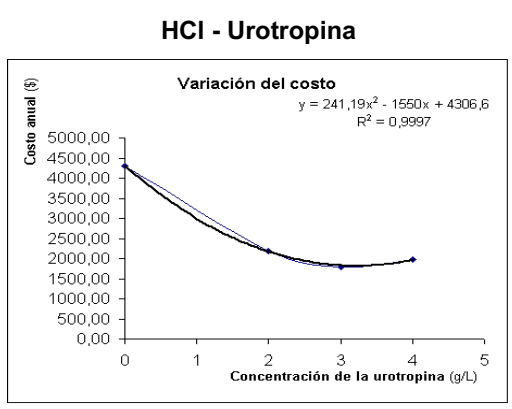

Concentración óptima de inhibidor: 3,21 g/L Costo mínimo de decapado: 1814,69 \$/año

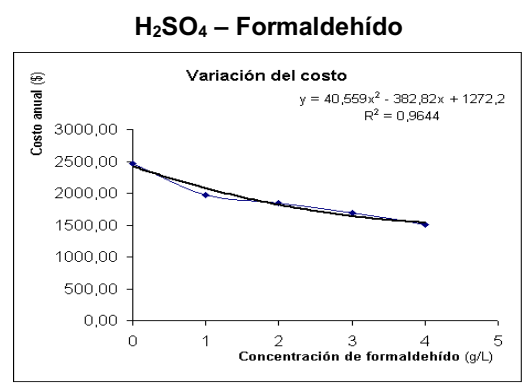

Concentración óptima de inhibidor: $4,72 \mathrm{~g} / \mathrm{L}$ Costo mínimo de decapado: 1519,14 \$/año

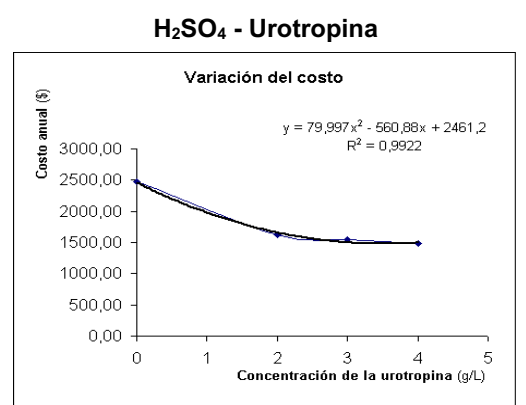

Concentración óptima de inhibidor: 3,51 g/L Costo mínimo de decapado: 1476,27 \$/año

Figura 1. Resultados de la optimización

Nótese que para el caso del ácido fosfórico con la urotropina hubo mucha dispersión en los resultados y el coeficiente de correlación del polinomio obtenido fue muy pequeño, por lo que este resultado no se utilizó en el experimento dirigido a comprobar el efecto del uso del inhibidor en la calidad de los recubrimientos

\section{DISCUSIÓN}

Como resultados del desarrollo experimental en la evaluación de inhibidores de corrosión en el decapado ácido se demostró el efecto inhibidor del formaldehído y la urotropina para los tres ácidos probados en las condiciones de operación del decapado que se realiza en el taller objeto de estudio. 
Combinando el $\mathrm{HCl}$ y el formaldehído, para las cuatro concentraciones de inhibidor evaluadas, la mejor combinación resultó ser $3 \mathrm{~g} / \mathrm{L}$, con la que se redujo en 2,66 veces la velocidad de corrosión desde un valor de 51,89 mm/año hasta 19,46 mm/año, con una eficiencia inhibidora del $62,5 \%$, siendo esta mayor que la alcanzada con urotropina a $3 \mathrm{~g} / \mathrm{L}$ que resultó ser 59,37 \%, disminuyendo la velocidad de corrosión 2,46 veces, desde un valor de 51,89 mm/año hasta $21,08 \mathrm{~mm} / \mathrm{año}$.

Las velocidades de ataque a la superficie metálica con los ácidos fosfórico y sulfúrico son mucho más grandes que con el ácido clorhídrico ya que estos fueron evaluados a $80^{\circ} \mathrm{C}$ como recomienda la norma NC 12-03-01: 1981 y no a temperatura ambiente como el caso del $\mathrm{HCl}$.

El $\mathrm{H}_{2} \mathrm{SO}_{4}$ combinado con formaldehído disminuye 5,38 veces la velocidad de corrosión para la mejor combinación probada a $4 \mathrm{~g} / \mathrm{L}$, desde 2689,04 mm/año hasta 499,10 mm/año, con una eficiencia inhibidora de 81,43\%. La urotropina con este mismo ácido y a la misma concentración de inhibidor ofrece un coeficiente de protección de 6,64, con una disminución de la velocidad de corrosión desde 2689,04 mm/año hasta 405,00 mm/año, reportando una eficiencia inhibidora del 84,93\%.

El $\mathrm{H}_{3} \mathrm{PO}_{4}$ combinado con formaldehído arroja una eficiencia inhibidora muy baja con un valor de $44 \%$ para la mejor combinación probada a $3 \mathrm{~g} / \mathrm{L}$ de inhibidor, y una disminución de la velocidad de corrosión solo de 1,808 veces, desde 1742,41 mm/año hasta 963,92 mm/año. Con la urotropina no se aprecia efecto inhibidor significativo e igualmente no se observa una disminución de la velocidad proporcional al aumento de la concentración del inhibidor, esto lo demuestra el comportamiento de las curvas de costo contra concentración del inhibidor, que mientras que para las otras combinaciones el modelo se ajustó a un polinomio de segundo orden con coeficientes de regresión mayores de 0,95 , para este caso no se encontró ninguna línea de tendencia ajustable (ver la Figura 1).
Con el análisis de estas curvas y de los resultados obtenidos de velocidad de corrosión, eficiencia inhibidora y coeficiente de protección, se comprobó que el inhibidor mejora su efecto a medida que aumenta su concentración hasta un valor óptimo, ya que después de este valor, aunque se aumente su concentración, no continúa disminuyendo la velocidad de corrosión e incluso, puede revertir su efecto convirtiéndose en un catalizador, llegando a ser perjudicial en el proceso como es el caso del HCL con la Urotropina y del $\mathrm{H}_{3} \mathrm{PO}_{4}$ con Formaldehído.

Al introducir a escala industrial el uso del $\mathrm{HCl}$, $\mathrm{H}_{3} \mathrm{PO}_{4}$ y $\mathrm{H}_{2} \mathrm{SO}_{4}$ con la combinación de sus respectivos inhibidores a la concentración óptima, se comprobó que la utilización de estas sustancias no provoca ninguna afectación en la calidad de los recubrimientos, ya que los mismos cumplen con las normas, parámetros y exigencias técnicas del proceso, lo cual fue avalado por los especialistas de control de calidad del taller.

Los resultados indican que la combinación del ácido sulfúrico con cualquiera de los inhibidores es más ventajosa que los decapados con los otros ácidos, a pesar del alto costo del vapor en la entidad y el empleo de este ácido a $80^{\circ} \mathrm{C}$, esto está dado por el mayor precio de los otros ácidos y por la baja relación molar respecto al metal en la reacción de corrosión para el sulfúrico. Dado que la evaluación de los inhibidores se ha realizado solamente para un tipo de acero (SAE 1045), debe resultar necesario realizar evaluaciones similares para otros tipos de acero y otros metales y aleaciones. Para esos casos, pudiera aplicarse la metodología aplicada en este trabajo.

\section{CONCLUSIONES}

La introducción del formaldehído y la urotropina como inhibidores de corrosión en la operación de decapado ácido es factible en las condiciones del taller y atendiendo a las recomendaciones de las normas técnicas vigentes sobre los procesos de decapado, resultando mejor la combinación del ácido sulfúrico con cualquiera de los dos inhibidores. En cada uno de estos casos el costo del proceso de decapado ácido es menor que 
para los otros ácidos empleados en este estudio. Desde el punto de vista medio ambiental, la introducción de los inhibidores y los resultados de esta investigación traerá consigo una disminución sensible de las emisiones de gases (fundamentalmente de hidrógeno) en la zona de trabajo, dado por el menor ataque que sufren las piezas. Esto disminuye también el volumen de residual líquido ácido que se genera, al prolongar el periodo de cambio de baños agotados y, al proteger las piezas, reduce la posibilidad de la pérdida de vida útil de las mismas con el ahorro de metal.

\section{REFERENCIAS BIBLIOGRÁFICAS}

Boizán, M. A. (1988). Optimización (M. G. Ascanio Ed.).

Buralla, E. (2012). Estudio del efecto de los inhibidores de armaduras del hormigón. (Master), Universidad Politécnica de Valencia, Valencia. España. Disponible en: https://riunet. upv.es/bitstream/handle/10251/17809/ ESTUDIO\%2 ODEL\%2 OEFECTO\%20 DE \% 2 O LOS \% 20 INHIBIDORE S \% 20 DE\%20LA\%20CORROSI\%C3\%93N\%20 E N \% 20 A R M A DURA S \% 20 D E L \% 20 HORMIG\%C3\%93N.pdf?sequence=1

Camejo, J. J., y Domínguez, J. A. (2015). Métodos químico-tecnológicos de protección contra la corrosión. In. Disponible en: https:// www.researchgate.net/profile/JuanCamejo-Giniebra/publication/323176272_ Metodos_quimico-tecnologicos_ de_proteccion_contra_la_corrosion/ links/5a88be28a6fdcc6b 1 a 3f8889/ Metodos-quimico-tecnologicos-deproteccion-contra-la-corrosion.pdf

Carrillo, J. (2014). Estudio de la recuperación del zinc presente en los baños agotados de decapado procedentes de las industrias de galvanizado de zinc en caliente mediante técnicas electroquímicas. (Doctorado), Universidad Politecnica de Valencia,
Valencia, españa.Disponible:https://riunet. upv.es/bitstream/handle/10251/39370/ Carrillo\%20-\%20Estudio\%20de\%20la\%20 recuperaci\%F3n\%20del\%20zinc\%20 presente\%20en\%20los\%20ba\%F1 os\%20 agota

CEN. (1981). NC. 12-03-01.1981. SISTEMA DE NORMAS PARA LA PROTECCION CONTRA LA CORROSION. Preparacion de las superficies metalicas para la aplicacion de los recubrimientos. Requisitos generales. In (pp. 20). La Habana: Unidad poligráfica CEN.

CEN. (1987). NC. 12-04-39.1987. INHIBIDORES DE CORROSION ACIDA. Métodos de determinación de la capacidad protectora durante el decapado ácido de metales. In (pp. 14). La Habana: Unidad poligráfica CEN.

Domínguez, J. A., Castro, D. M., Matos, R., y Hing, R. (1987). Introducción a la corrosión y protección de metales. Habana: Ministerio de Educación Superior.

Pizarro, R. (2006). Protección contra la corrosión mediante el empleo de inhibidores. Revista peruana de química e ingeniería química, 9(1), 25-32. Disponible en: https:// revistasinvestigacion.unmsm.edu.pe/index. php/quim/article/view/4043/3220

Prado, D. R. (2015). Productos naturales con efectos anticorrosivos y generadores de copolímeros. (Ingeniería), Universidad Central del Ecuador, Quito,Ecuador. Disponible en: http://www.dspace.uce.edu. ec/handle/25000/3770

Uhlig, H. H., y Revie, R. W. (2008). Corrosion And Corrosion Control. An Introduction To Corrosion Science And Engineering (E. J. W. a. Sons Ed. Fourth Edition ed.). Hoboken New Jersey 